This document is confidential and is proprietary to the American Chemical Society and its authors. Do not copy or disclose without written permission. If you have received this item in error, notify the sender and delete all copies.

\title{
Towards Artificial Mitochondrion: Mimicking Oxidative Phosphorylation in Polymer and Hybrid Membranes
}

\begin{tabular}{|c|c|}
\hline Journal: & Nano Letters \\
\hline Manuscript ID & nl-2017-030935 \\
\hline Manuscript Type: & Communication \\
\hline Date Submitted by the Author: & 20-Jul-2017 \\
\hline Complete List of Authors: & $\begin{array}{l}\text { Otrin, Lado; Max-Planck-Institut fur Dynamik komplexer technischer } \\
\text { Systeme, Process System Engineering } \\
\text { Marušič, Nika; Max-Planck-Institut fur Dynamik komplexer technischer } \\
\text { Systeme, Process System Engineering } \\
\text { Bednarz, Claudia; Max-Planck-Institut fur Dynamik komplexer technischer } \\
\text { Systeme, Process System Engineering } \\
\text { Vidaković-Koch, Tanja; Max-Planck-Institut fur Dynamik komplexer } \\
\text { technischer Systeme, Process System Engineering } \\
\text { Landfester, Katharina; Max Planck Institute for Polymer Research, } \\
\text { Sundmacher, Kai; Max Planck Institute for Dynamics of Complex Technical } \\
\text { Systems, Process Engineering }\end{array}$ \\
\hline
\end{tabular}




\title{
Towards Artificial Mitochondrion: Mimicking
}

\section{Oxidative Phosphorylation in Polymer and Hybrid}

\author{
Membranes \\ Lado Otrin,,$^{\dagger}$ Nika Marušič, Claudia Bednarz,,$^{\dagger}$ Tanja Vidaković-Koch, ${ }^{\dagger,}$ Katharina Landfester, \\ and Kai Sundmacher ${ }^{\dagger}, \S$ \\ ${ }^{\dagger}$ Max Planck Institute for Dynamics of Complex Technical Systems, Sandtorstrasse 1, 39106 \\ Magdeburg, Germany \\ Max Planck Institute for Polymer Research, Ackermannweg 10, 55128 Mainz, Germany \\ ${ }^{\S}$ Otto von Guericke University, Universitaetsplatz 2, 39106 Magdeburg, Germany \\ * Corresponding author: vidakovic@mpi-magdeburg.mpg.de, +49 3916754630
}

For energy supply to biomimetic constructs, a complex chemical energy-driven ATP-generating artificial system was built. The system was assembled with bottom-up detergent-mediated reconstitution of an ATP synthase and a terminal oxidase into two types of novel nanocontainers, built from either graft copolymer membranes or from hybrid graft copolymer/lipid membranes. The versatility and biocompatibility of the proposed nanocontainers was demonstrated through convenient system assembly and through high retained activity of both membrane-embedded enzymes. In future, the nanocontainers might be used as a platform for the functional 
reconstitution of other complex membrane proteins and could considerably expedite the design of nanoreactors, biosensors and artificial organelles.

Keywords: Nanoreactors, nanocontainers, energy regeneration, artificial organelle, hybrid materials, bottom-up.

Bottom-up synthetic biology offers a unique opportunity of combining man-made and biological parts, for creating augmented biological systems which as such do not exist in nature. ${ }^{1,2}$ The combination of synthetic polymers and biological components, such as integral membrane proteins (IMPs), appears especially appealing since polymers provide excellent mechanical stability and robustness, while the proteins introduce their intrinsic functionalities. ${ }^{3}$ Some utilization of similar artificial systems, comprised of functionalized polymer containers, was seen over the last decade in medicine and pharmacology as drug delivery systems ${ }^{4-6}$ and ${\text { contrasting } \text { agents }^{7-9} \text { as well as in biotechnology as nanoreactors }}^{10-12}$ and biosensors ${ }^{13,14}$. However, for several applications (drug delivery, construction of artificial organelles etc.), functionalization of polymer containers with more complex, environment-sensitive membrane proteins, is a necessity.

At this point, predominantly very rigid proteopolymersomes formation methods seem to vastly limit the set of IMPs suitable for functional reconstitution to the few robust, most stable, simple model IMPs such as ompF, Tsx, AQP0 and AqpZ. ${ }^{15-18}$ Meanwhile, more and more IMPs had been successfully reconstituted into liposomes each year and more complex systems, comprised of two or more co-reconstituted IMPs had been formed. ${ }^{19-21}$ Reconstitutions of IMPs into liposomes are in the majority of cases mediated by the use of detergents. A possibility to apply similar detergent-based reconstitution methods, optimized for high performance of the 
reconstituted membrane protein, to suitable polymer or hybrid membranes, would considerably expedite the formation of new versatile nanoreactors, biosensors and complex artificial systems.

A first step towards the integration of more complex IMPs into polymersomes was made by Choi and Montemagno by the integration of bacteriorhodopsin in the form of purple membranes along with ATP synthase into polymersomes, using the triblock copolymer PEtOz-PDMSPEtOz. $^{22}$ The light-driven synthesis of ATP was successfully demonstrated in this multiprotein polymersome system and it was attributed to the biocompatibility of the tested polymer. However, no evidence presented in this study suggested that the observed functionality of ATP synthase was not instead facilitated by the large amount of lipids introduced to the polymersomes in the form of purple membranes.

A recent example, that clearly demonstrated how the functionality of the environment-sensitive membrane protein was sacrificed for higher overall stability and durability of the artificial system, was the one of cytochrome $\mathrm{bo}_{3}$ quinol oxidase ( $\mathrm{bo}_{3}$ oxidase) reconstituted in diblock copolymer PBd-PEO containers as well as in more complex hybrid PBd-PEO/POPC containers. ${ }^{23}$ While the bo $_{3}$ oxidase retained most of its activity in hybrid containers featuring high lipid content (50-75\%), a staggering $70 \%$ reduction of activity was observed in hybrid containers at low lipid content $(25 \%)$. Furthermore, $\mathrm{bo}_{3}$ oxidase reconstituted in pure polymersomes showed no significant activity, exposing the poor biocompatibility of the PBdPEO membranes. Nevertheless, this study revealed the high relevance of hybrid lipid/polymer containers to the emerging field of synthetic biology, for which the stability and durability of the artificial systems are as important as the high retained functionality of all membrane-embedded components of the system. 
In this Letter we take a step away from the commonly used block copolymer-based nanocontainers and introduce versatile, stable, biocompatible and immunostealth ${ }^{24}$ nanocontainers based on the graft copolymer poly(dimethylsiloxane)-graft-poly(ethylene oxide) (PDMS- $g$-PEO). In aqueous solutions this polymer self-assembles into stable vesicular structures ${ }^{25}$ with a reported membrane core thickness of $5 \mathrm{~nm} \cdot{ }^{26}$ Due to the polymer membrane thickness similar to the one of a typical lipid bilayer and its high fluidity of $360 \mathrm{cSt}$, PDMS-gPEO is a promising substitute for classical lipid membranes. Furthermore, PDMS-g-PEO can be mixed with lipids to form hybrid lipid/polymer membranes with the composition-dependent, predictable homogeneous or heterogeneous (phase-separated) distribution of both components in the membranes. ${ }^{27}$ Conveniently, polymer or hybrid vesicles with controlled mean diameters can easily be obtained by successive extrusion (SUVs, LUVs), while larger vesicles (GUVs), suitable for vesicle characterization, can be produced using the electroformation procedure.

An artificial system, capable of chemical energy-driven ATP production (schematically represented in Fig. 1C) was established through bottom-up detergent-mediated integration of purified transmembrane proteins, the $\mathrm{bo}_{3}$ oxidase and the $\mathrm{F}_{1} \mathrm{~F}_{\mathrm{O}}$ ATP synthase, into the proposed novel nanocontainers. In parallel, it's optimized for performance natural counterpart $\left(\mathrm{bo}_{3}\right.$ oxidase/ATP synthase proteoliposomes) was adapted as a benchmark for the evaluation of biocompatibility of our nanocontainers. The $\mathrm{bo}_{3}$ oxidase, a chemical energy-driven proton pump, was used to establish a proton gradient, sufficiently high to drive the synthesis of ATP. The prerequisites for the successful ATP synthesis in this system were: 1) a functionally reconstituted, active $\mathrm{bo}_{3}$ oxidase with the quinol binding site well accessible to ubiquinol-1 $\left(\mathrm{Q}_{1}\right.$, the electron shuttle), 2) a tight, proton-impermeable membrane and 3) a membrane capable of facilitating the rotational motion of ATP synthase as well as the proton translocation by both 
enzymes. Thickness and fluidity of the artificial membranes in particular are expected to play a crucial role in functional reconstitution of the described system. Finally, the integration of the described system was established through a detergent-mediated reconstitution. The versatility of the polymer and hybrid nanocontainers in regard to system's assembly was demonstrated with detergents, commonly used for the reconstitution of IMPs into liposomes, namely sodium cholate (SC), sodium deoxycholate (SDC) and octyl $\beta$-D-glucopyranoside (OG).

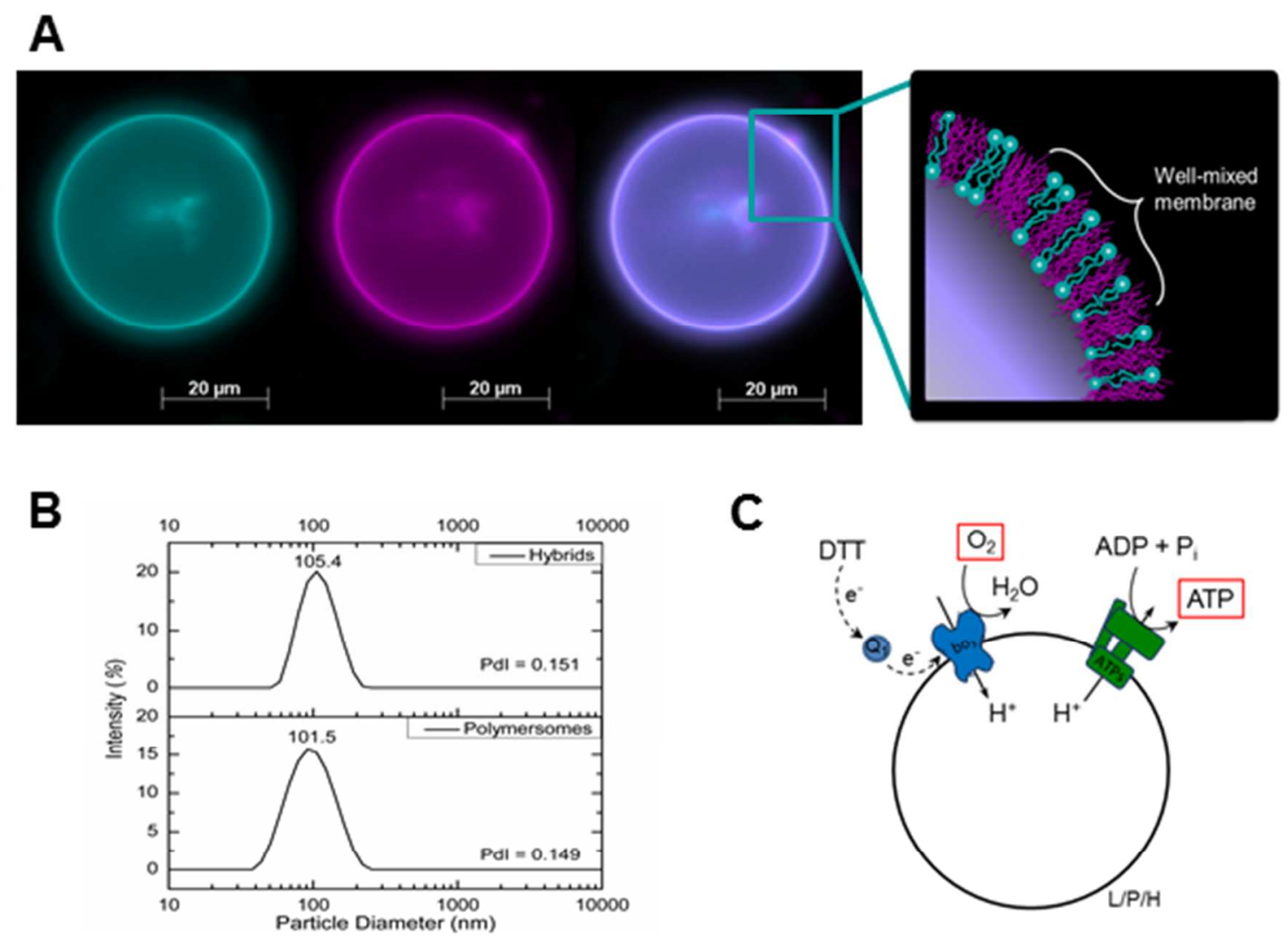

Figure 1. (A) Fluorescence microscopy images of homogeneous hybrid microcontainer comprised of $70 \mathrm{~mol} \%$ PDMS-g-PEO and $30 \mathrm{~mol} \%$ of soy PC. The distribution of lipid (left) and polymer (middle) is homogeneous in well-mixed (right) hybrid membranes. (B) Intensity-based size distribution of hybrid (top) and polymer (bottom) nanocontainers after the extrusion, with their corresponding polydispersity indexes indicated in the bottom-right corner of each plot. (C) 
Schematic representation of the ATP generating system. Measured variables, used for the determination of the activity of both enzymes in lipid (L), polymer (P) or hybrid (H) nanocontainers are marked with red squares.

For the purpose of characterization, first micron-sized hybrid containers were prepared with the electroformation procedure from, for the first time, natural lipid extract soy phosphatidylcholine (soy PC) and PDMS-g-PEO, in various polymer-to-lipid ratios. To enable observation of both components of the nanocontainers, the fluorescein-labelled polymer probe was synthesized (PDMS-g-PEO-fluo) and the distribution of both components in the artificial membranes was analyzed by use of fluorescence microscopy. Homogeneous, well-mixed membranes were observed at and above $70 \mathrm{~mol} \%$ of polymer in the membranes (Fig. 1A), while phase-separated membranes were observed at lower polymer concentrations (SI Fig. 1). Homogeneous hybrid microcontainers were stable for at least several weeks. Similar results were reported for polymer/synthetic POPC hybrid microcontainers. ${ }^{27}$ In the following experiments, homogeneous hybrid nanocontainers intended for later system integration were prepared with a polymer-to-lipid mol\% ratio of $75: 25$, featuring the highest stability in the case of hybrid microcontainers.

For the reconstitution of both membrane proteins, nano-sized polymer containers as well as homogeneous hybrid containers were produced with the extrusion technique. Intensity-based size distribution and polydispersity of nanocontainers were tracked by dynamic light scattering (DLS). Both types of nanocontainers were uniform in size (Fig. 1B) with Z-averages (105 nm for hybrids and $102 \mathrm{~nm}$ for polymersomes) being close to the diameter of pores of the extrusion filter $(100 \mathrm{~nm})$. A low polydispersity index, very similar to the one of liposomes, was obtained for both types of nanocontainers. 
In the next step, the activity of the $\mathrm{bo}_{3}$ oxidase reconstituted in hybrid and polymer nanocontainers (reconstitution described in the SI) was monitored. The changes in oxygen concentration during a turnover of the $\mathrm{bo}_{3}$ oxidase were detected with the Clark-type electrode (Fig. 2A). The $\mathrm{bo}_{3}$ oxidase was reconstituted with all tested detergents and the activities were compared to the activities of the enzyme in liposomes (SI Fig. 2 and SI Fig. 3).
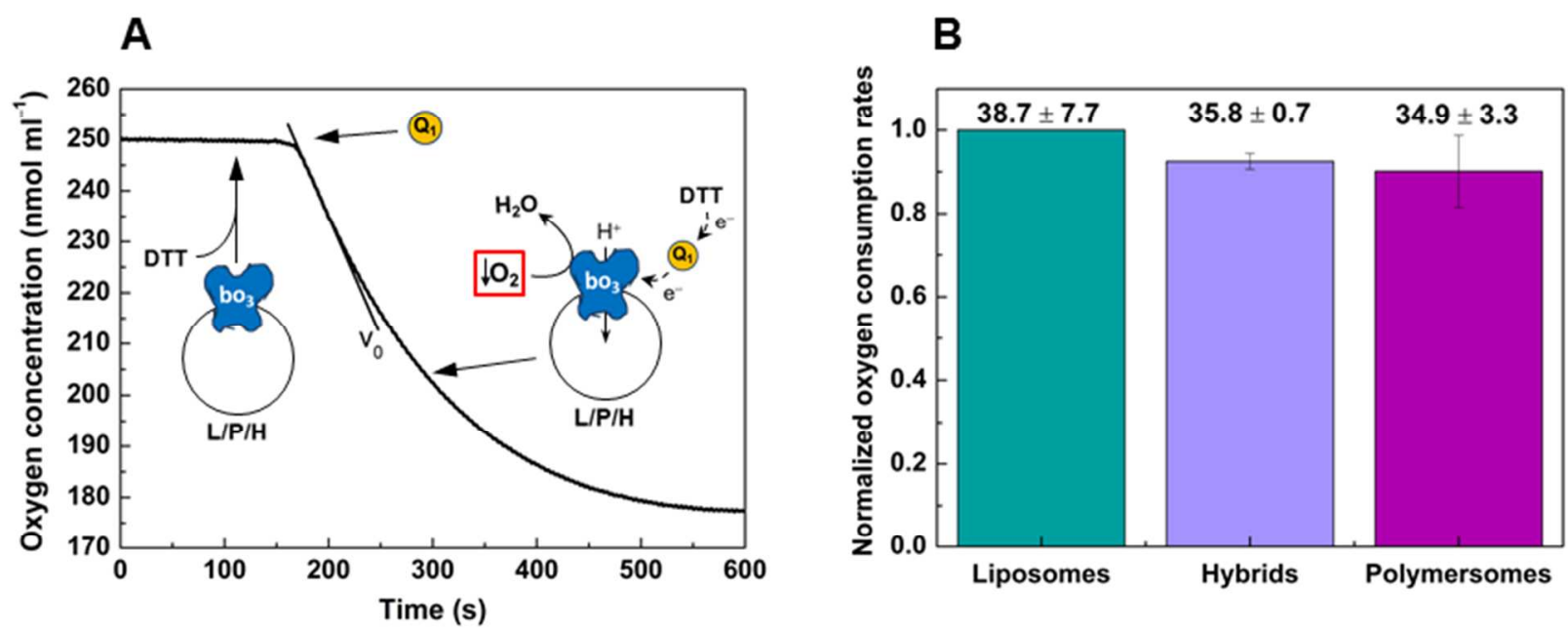

Figure 2. (A) Changes in oxygen concentration due to the activity of $\mathrm{bo}_{3}$ oxidase in hybrid nanocontainers. At $t \approx 170 \mathrm{~s}$ mark, the reaction was initiated by ubiquinone- $1\left(\mathrm{Q}_{1}\right)$. The trace shown is for the hybrid nanocontainers with membrane-embedded enzyme reconstituted with 0.2 $\%$ SDC. The oxygen consumption rate was determined from the slope indicated as " $\mathrm{v}_{0}$ ". (B) The comparison of highest obtained oxygen consumption rates for the natural and two artificial systems with the corresponding values expressed in nmol of $\mathrm{O}_{2} \mathrm{ml}^{-1} \mathrm{~min}^{-1}$.

With the measured oxygen consumption rates as high as $35.8 \mathrm{nmol} \mathrm{ml}^{-1} \mathrm{~min}^{-1}$ (hybrids) and $34.9 \mathrm{nmol} \mathrm{ml}{ }^{-1} \mathrm{~min}^{-1}$ (polymersomes), we were able to prove the successful functional integration of $\mathrm{bo}_{3}$ oxidase into both types of nanocontainers. Compared to proteoliposomes (Fig. 2B) only a minimal loss of activity was observed. Having in mind previous reports ${ }^{23}$ on $b_{3}$ oxidase reconstitution in polymersomes and hybrid vesicles, where the enzyme was completely 
deactivated upon integration in polymersomes and retained only $30 \%$ of its original activity in hybrid vesicles, the activity of $\mathrm{bo}_{3}$ oxidase found in the present study, in both hybrid and polymer vesicles, is truly remarkably high.
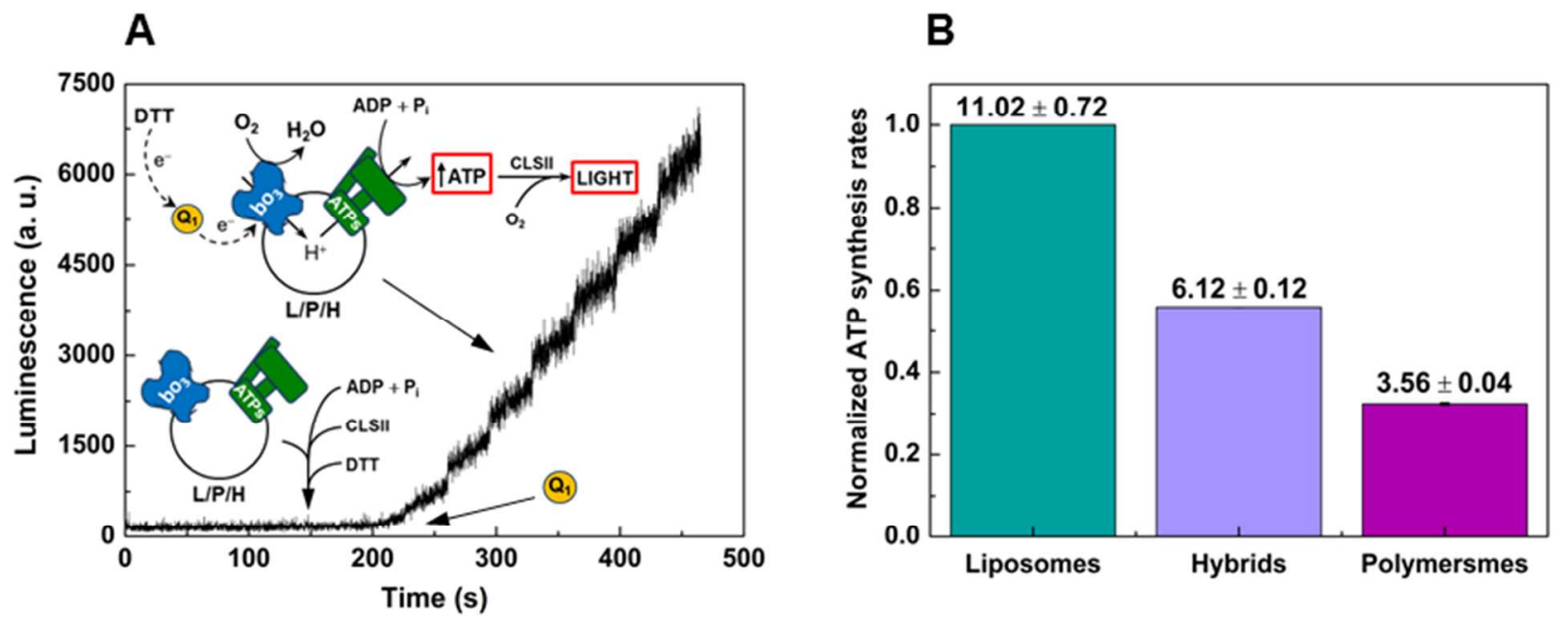

Figure 3. (A) Changes in luminescence generated by luciferin/luciferase system as a result of respiration-driven ATP synthesis. Proton-pumping by the $\mathrm{bo}_{3}$ oxidase was initiated at $t=200 \mathrm{~s}$ mark by the addition of ubiquinone- $1\left(\mathrm{Q}_{1}\right)$. The slope normalized against a known amount of ATP represents the ATP synthesis rate. The trace shown is for the energy regeneration system reconstituted in hybrid nanocontainers with $0.05 \%$ OG. (B) The comparison of highest obtained ATP synthesis rates for natural, hybrid and polymer-based artificial system with the corresponding values, expressed in molecules of ATP/ATP synthase/s.

The complete artificial system was assembled with octyl glucoside, the detergent which was shown to reconstitute highly active $\mathrm{bo}_{3}$ oxidase. Respiration-driven ATP synthesis rates of proteonanocontainers were determined based on bioluminescence produced by the luciferin/luciferase CLSII reagent ${ }^{28}$ (Fig. 3A). A comparison of the highest achieved ATP synthesis rates of the natural, hybrid and polymer-based artificial systems are depicted in Fig. 3B (for the comparison of ATP synthesis rates of hybrid and polymer nanocontainers see SI Fig. 4). 
An overall highest ATP synthesis rate of 11 ATP/ATP synthase/s was observed in liposomes, reconstituted with $0.6 \%$ SC. Interestingly, the highest ATP synthesis rates of proteohybrid and polymer nanocontainers of 6.1 ATP/ATP synthase/s and 3.6 ATP/ATP synthase/s, respectively, were observed at very low concentrations of OG (0.05 and $0.1 \%$ OG) for both types of nanocontainers. Due to the relatively high cost of detergents, the nanocontainers, presented in this Letter might be considered an attractive, cost-efficient alternative to liposomes. The artificial energy regeneration system was able to continuously produce ATP for at least several hours at $6 \times$ higher ATP synthesis rate than a similar, light-driven system ${ }^{22}$, reconstituted in hybrid nanocontainers. With the sufficient supply of co-factors to the artificial system, physiological levels of the produced ATP can be reached, which is a prerequisite for a large number of biological and biomimetic processes. While we believe that the co-reconstitution of ATP synthase with another membrane protein into artificial containers, presented in this study, could certainly be improved further, we conclude that the achieved high ATP synthesis rates of this complex system are a good indicator of the excellent biocompatibility of the proposed novel nanocontainers.

In this study we presented a new type of stable, versatile, stealth and fully biocompatible nanocontainers based on graft copolymer PDMS-g-PEO. The copolymer alone was used for the formation of polymersomes or was combined with natural lipid extract for the formation of hybrid lipid/polymer nanocontainers. We demonstrated an excellent uniformity of both types of nanocontainers, reflected in consistent size, low polydispersity and a good control over the distribution of both components in hybrid membranes. The presented nanocontainers offer a versatile platform for the reconstitution of various new and complex membrane proteins. $\mathrm{Bo}_{3}$ oxidase functionalized hybrid nanocontainers could serve as $\mathrm{pH}$-switchable encapsulated 
substrate-processing nanoreactors. Additionally, PDMS-g-PEO polymersomes, functionalized with the desired IMPs, may also show great potential as stealth drug delivery systems. Unlike commonly used block copolymer-based delivery systems, graft copolymer-based nanocontainers can readily be dissolved to release the encapsulated substance.

With the bottom-up approach and through the employment of commonly used detergents, we integrated two purified enzymes into both types of nanocontainers to form complex, respirationdriven ATP-generating artificial systems. High activity of both integrated enzymes further indicated excellent biocompatibility as well as integrity of the artificial membranes. The presented artificial system, capable of continuous production of ATP, shows great promise in the exciting, rapidly developing field of synthetic biology, where it could be used to supply energy to countless natural and synthetic energy-demanding biomimetic constructs.

\title{
Supporting Information Available:
}

Text describing materials and methods, figures SI Fig. 1, SI Fig. 2, SI Fig. 3 and SI Fig. 4. (PDF) The following files are available free of charge at http://pubs.acs.org.

\author{
ACKNOWLEDGMENT \\ This work is part of the MaxSynBio consortium which is jointly funded by the Federal Ministry \\ of Education and Research (BMBF) of Germany and the Max Planck Society. The authors are \\ grateful to Prof. C. von Ballmoos and his team from the University of Bern for the generous gift \\ of two plasmids used in this study and for invaluable suggestions and discussion.
}




\section{REFERENCES}

(1) Schwille, P. Science 2011, 333, 1252-1254.

(2) Channon, K.; Bromley, E. H. C.; Woolfson, D. N. Curr. Opin. Struct. Biol. 2008, 18, 491498.

(3) Discher, D. E.; Eisenberg, A. Science 2002, 297, 967-973.

(4) Moghimi, S. M.; Hunter, A. C.; Murray, J. C. FASEB J. 2005, 19, 311-330.

(5) Duncan, R. Nat. Rev. Drug Discovery 2003, 2, 347-360.

(6) Li, D.; Li, C.; Wan, G.; Hou, W. Colloids Surf., A 2010, 372, 1-8.

(7) Wang, M.; Zhang, M.; Siegers, C.; Scholes, G. D.; Winnik, M. A. Langmuir 2009, 25, 13703-13711.

(8) Yang, F.; Li, Y.; Chen, Z.; Zhang, Y.; Wu, J.; Gu, N. Biomaterials 2009, 30, 3882-3890.

(9) Ghoroghchian, P. P.; Therien, M. J.; Hammer, D. A. Wiley Interdiscip. Rev.: Nanomed. Nanobiotechnol. 2009, 1, 156-167.

(10) Ranquin, A.; Versées, W.; Meier, W.; Stayaert, J.; Van Gelder, P. Nano Lett. 2005, 5, 2220-2224.

(11) Broz, P.; Driamov, S.; Ziegler, J.; Ben-Haim, N.; Marsch, S.; Meier, W.; Hunziker, P. Nano Lett. 2006, 6, 2349-2353.

(12) Axthelm, F.; Casse, O.; Koppenol, W. H.; Nauser, T.; Meier, W.; Palivan, C. G. J. Phys. Chem. B 2008, 112, 8211-8217. 
(13) Gonzalez-Perez, A.; Stibius, K. B.; Vissing, T.; Nielsen, C. H.; Mouritsen, O. G. Langmuir 2009, 25, 10447-10450.

(14) Gibbs, J. M.; Park, S.-J.; Anderson, D. R.; Watson, K. J.; Mirkin, C. A.; Nguyen, S.T. J. Am. Chem. Soc. 2005, 127, 1170-1178.

(15) Klara, S. S.; Saboe, P. O.; Sines, I. T.; Babaei, M.; Chiu, P.-L.; DeZorzi, R.; Dayal, K.; Walz, T.; Kumar, M.; Mauter, M. S. J. Am. Chem. Soc. 2016, 138, 28-31.

(16) De Vocht, C.; Ranquin, A.; Willaert, R.; Van Ginderachter, J. A.; Vanhaecke, T.; Rogiers, V.; Versées, W.; Van Gelder, P.; Steyaert, J. J. Control. Release 2009, 137, 246-254.

(17) Stoenescu, R.; Graff, A.; Meier, W. Macromol. Biosci. 2004, 4, 930-935.

(18) Kumar, M.; Grzelakowski, M.; Zilles, J.; Clark, M.; Meier, W. Proc. Natl Acad. Sci. U.S.A. 2007, 104, 20719-20724.

(19) Jabůrek, M.; Costa, A. D. T.; Burton, J. R.; Costa, C. L.; Garlid, K. D. Circ. Res. 2006, 99, 878-883.

(20) Nordlung, G.; Brzezinski, P.; von Ballmoos, C. Nat. Commun. 2014, 5, 4303.

(21) von Ballmoos, C.; Biner, O.; Nilsson, T.; Brzezinski, P. Biochim. Biophys. Acta 2016, $1857,321-323$.

(22) Choi, H.-J.; Montemagno, C. D. Nano Lett. 2005, 5, 2538-2542.

(23) Khan, S.; Li, M.; Muench, S. P.; Jeuken, L. J. C.; Beales, P. A. Chem. Commun. 2016, 52, 11020-11023. 
(24) Jukarainen, H. J.; Clarson, S. J.; Seppälä, J. V.; Retzinger, G. S.; Ruohonen, J. K. Silicon 2012, 4, 231-238.

(25) Carlsen, A.; Glaser, N.; Le Meins, J.-F.; Lecommandoux, S. Langmuir 2011, 27, 48844890.

(26) Lin, Z.; Hill, R. M.; Davis, H. T.; Scriven, L. E.; Talmon, Y. Langmuir 1994, 10, 1008 1011.

(27) Chemin, M.; Brun, P.-M.; Lecommandoux, S.; Sandre, O.; Le Meins, J.-F. Soft Matter 2012, 8, 2867-2874.

(28) Strehler, B. L. Methods Biochem. Anal. 1968, 16, 99-181. 


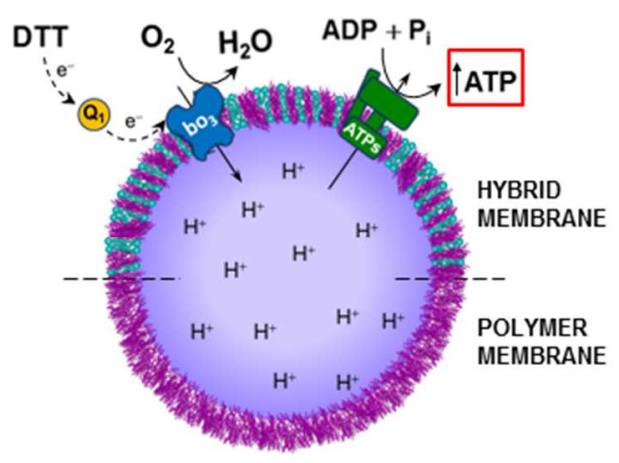

For Table of Contents Only. 